\title{
Interaction of Nutrition and Exercise on Bone and Muscle
}

\author{
Faidra Laskou and Elaine Dennison \\ MRC Lifecourse Epidemiology Unit, University of Southampton, Southampton General Hospital, Southampton, UK
}

DOI: https://doi.org/10.17925/EE.2019.15.1.11

$\mathrm{P}$ hysical activity and nutritional factors, such as calcium and vitamin D intake, have been shown in numerous studies to be beneficial to musculoskeletal health at different points in the lifecourse. However, the evidence that physical activity and nutrition may act synergistically for benefit is far more sparce; the best data come from studies that consider calcium, vitamin D or creatine supplementation in combination with physical activity interventions to promote better musculoskeletal health. Some observational data also suggest that a 'healthier' lifestyle, characterised by a more 'prudent' diet and higher reported levels of physical activity is also associated with better musculoskeletal health in late adulthood. Given the public health burden of osteoporosis and sarcopenia in our aging population, well designed randomised controlled trials are now timely and much needed.

\section{Keywords}

Bone, muscle, activity, nutrition, lifecourse

Disclosure: Faidra Laskou and Elaine Dennison have nothing to declare in relation to this article. Review Process: This article is a short opinion piece and has not been through the journal's standard peer review process, but has been reviewed by the Editorial Board for accuracy before publication.

Authorship: The named authors meet the criteria of the International Committee of Medical Journal Editors for authorship for this manuscript, take responsibility for the integrity of the work as a whole and have given final approval for the version to be published.

Compliance with Ethics: This article is an

opinion piece and does not report on new clinical

data, or any studies with human or animal

subjects performed by any of the authors.

Received: 1 December 2018

Accepted: 8 February 2019

Citation: European Endocrinology. 2019;15(1):11-2

Corresponding Author: Elaine Dennison,

MRC Lifecourse Epidemiology Unit, University of

Southampton, Southampton General Hospital,

Southampton SO16 6YD, UK. E: emd@mrc.soton.ac.uk

Support: No funding was received in

the publication of this article.
As the population ages, the need to promote good musculoskeletal health throughout the lifecourse increases. Physical activity and adequate nutrition are known to be beneficial to bone and muscle at all ages; individually the benefits of physical activity and inadequate or excessive nutrition on bone and muscle have been extensively researched. It is well recognised that physical activity and nutrition in childhood and adolescence contribute to bone and muscle development. ${ }^{1}$ These factors may have a direct influence or act indirectly through effects on linear growth and body composition. A study of 4-year-olds enrolled in the Southampton Women's Survey, mean daily time in moderate-vigorous physical activity and daily calcium intake at 3 years were positively related to hip bone size and density. ${ }^{2}$ At the opposite end of the lifecourse, preventive measures, including nutritional optimisation, food supplements and regular resistance-training physical exercise, are widely recommended in elderly subjects and may improve musculoskeletal heath by reducing or delaying the development of muscle wasting and subsequent sarcopenia. ${ }^{3}$ A particular point of interest to clinicians and researchers is whether there may be interaction between nutrition and physical activity with regard to musculoskeletal health. Multiple dietary components may affect osteocyte function and therefore may have a synergistic effect on bone health when combined with a regime of physical activity, ${ }^{4}$ while the topic of possible interaction between nutrition and physical activity on muscle health was also the focus of a recent systematic review. ${ }^{5}$

A 'prudent' dietary pattern during adulthood is characterised by greater intake of low-fat milk and yogurts, whole-grain bread and breakfast cereals, fruits, and vegetables, and lower intake of sugars, sweets, processed foods and animal fats. ${ }^{6}$ Such a diet has been shown to be associated with better bone health in women, though no association has been found in men. ${ }^{6}$ Specifically, calcium intake has been extensively linked to bone health throughout the lifecourse, with evidence of benefit of adequate intakes throughout life, with other vitamins ( $K$ and $E$ ) also implicated for bone health. ${ }^{7}$ A healthier diet in later life has similarly been associated with lower risk of decline in physical performance in older adults. ${ }^{8}$ In a cohort of older UK adults, higher diet quality at age 60-64 was associated with faster chair rise speed and with longer balance time. ${ }^{8}$

With regard to physical activity, peak bone mass achieved in youth is the strongest predictor of later life osteoporosis risk. ${ }^{9}$ Sedentary behaviour has been shown to have a negative impact on bone health but the frequency of osteogenic activities rather than volume of physical activity appears most important. ${ }^{10}$ High-impact sporting activities are associated with higher levels of bone turnover in females. ${ }^{11}$ Cortical bone appears to be more sensitive to physical activity than cancellous bone in the peripheral skeleton..$^{12,13}$ The detrimental effect of sedentary behaviour on bone health could derive from lack of muscle activation and unloading of bone structures. Type and intensity of physical activity appear important; vertical accelerations and moderate-vigorous physical activity independently predict bone properties, especially at weight-bearing sites such as the tibia in older men and women. ${ }^{14}$ The importance of physical activity in promoting muscle health throughout the lifecourse seems intuitive; resistance exercise training is more effective in increasing muscle 
mass and strength, whereas endurance exercise training is superior for maintaining and improving maximum aerobic power. ${ }^{15}$

From a public health intervention standpoint, possible interaction between physical activity and nutrition is of particular interest. Researchers have demonstrated associations between levels of circulating 25-hydroxyvitamin D and moderate-vigorous physical activity, ${ }_{1}^{4}$ while another study has shown significant improvement in the Timed Up and Go test when vitamin D supplementation was added to the exercise programme. ${ }^{16}$ By contrast, supplementation with green tea, magnesium oxide, milk fat globule membrane, soy isoflavones, dairy and fruit products enhanced with vitamins and minerals, and tea catechin provided no added benefit in respect to their effect on muscle mass and/ or strength in other studies. ${ }^{17,18}$ Observational data from cohort studies suggest that lifestyle factors, such as physical activity and prudent diet score, may impact bone health..$^{19}$ In the Hertfordshire Cohort Study, in a cross-sectional study of 498 men and 498 women aged 59-72 years, information on physical activity, diet quality, history of prior fracture, family history of fracture and cigarette and alcohol consumption were obtained through a questionnaire. ${ }^{18}$ In women, a graded association between the number of risk factors and bone density at the lumbar spine and total femur was seen, with the strongest association seen in those with three or more risk factors. Furthermore, among women, strong graded relationships were seen between the number of risk factors and incident fracture.

Clearly, randomised controlled trials will be required to remove the possibility of confounding variables. A recent systematic review that considered the interaction between physical activity and nutrition showed that physical exercise has a positive impact on muscle mass and muscle function in people over 65 years old, but benefits of dietary supplementation appeared to be limited. ${ }^{5}$ The most positive finding was creatine supplementation, which was found to be beneficial in improving muscle mass and muscle strength and had an interactive effect on physical performance in one of five studies. A notable feature of this systematic review, which comprised 37 randomised controlled trials, was the heterogeneity with regard to physical exercise and dietary supplementation. Eighty percent of studies showed benefits on muscle mass with exercise training, and in the majority of cases where no benefit was observed these studies were undertaken in frail, elderly patients, possibly reflecting lower levels of activity in this group. With regard to possible synergy with protein supplementation in this study, the results follow a previous meta-analysis that showed a positive effect of protein supplementation on muscle mass gains during prolonged, resistance-type exercise training in older subjects. ${ }^{20}$

In conclusion, the subject of possible synergy between nutrition and physical activity in promoting musculoskeletal health throughout the lifecourse is an attractive one, but perhaps not a new concept. A previous excellent review by Daly and colleagues highlighted that although exercise and nutrition have contrasting influences on the tissue-level processes responsible for bone health, both can influence various circulating hormones, growth factors and inflammatory cytokines, which have been implicated in the pathophysiology of bone and muscle loss. ${ }^{19}$ To date, while the evidence for a beneficial effect of physical exercise on muscle mass, strength or physical performance is a compelling one, far fewer data are available to support a role for dietary supplementation for additional benefit. This most likely reflects the heterogenous nature of the study groups in the trials performed to date, and highlights a need for further well-designed studies that carefully document subjects' baseline exercise level and nutritional status. It is striking how little our knowledge base has increased since Daly's review in 2014, which focused on the benefits of combining physical activity and calcium intake on bone mass ${ }^{19}$ with some evidence found for the interaction of protein intake and exercise for muscle mass. The question of a threshold effect for physical activity and the possibility of other nutritional factors that may be key is a tantalising one that requires well-designed clinical trials to inform clinical practice. $\square$
1. Yang S, Nguyen ND, Center JR, et al. Association between abdominal obesity and fracture risk: A prospective study. J Clin Endocrinol Metab. 2013;98:2478-83.

2. Harvey NC, Cole ZA, Crozier SR, et al. Physical activity, calcium intake and childhood bone mineral: A population-based cross-sectional study. Osteoporos Int. 2012;23:121-30.

3. Ethgen O, Beaudart C, Buckinx F, et al. The future prevalence of sarcopenia in Europe: A claim for public health action. Calcif Tissue Int. 2017:100:229-34.

4. Willems HME, van den Heuvel EGHM, Schoemaker RJW, et al. Diet and exercise: A match made in bone. Curr Osteoporos Rep. 2017; 15:555-63

5. Beaudart C, Dawson A, Shaw SC, et al. Nutrition and physical activity in the prevention and treatment of sarcopenia: Systematic review. Osteoporos Int. 2017;28:1817-33.

6. Ward KA, Prentice A, Kuh DL, et al. Life course dietary patterns and bone health in later life in a British birth cohort study. J Bone Miner Res. 2016;31:1167-76.

7. Prentice A. Diet, nutrition and the prevention of osteoporosis Public Health Nutr. 2004;7:227-43.

8. Robinson SM, Westbury LD, Cooper R, et al. Adult lifetime diet quality and physical performance in older age:
Findings from a British birth cohort. J Gerontol Ser A. 2018;73:1532-7.

9. Weaver $\mathrm{CM}$, Gordon $\mathrm{CM}$, Janz KF, et al. The Nationa Osteoporosis Foundation's position statement on peak bone mass development and lifestyle factors: A systematic review and implementation recommendations. Osteoporos int. 2016;27:1281-386

10. Chastin SF, Mandrichenko O, Skelton DA. The frequency of osteogenic activities and the pattern of intermittence between periods of physical activity and sedentary behaviour affects bone mineral content: The cross-sectional NHANES study. bone mineral content: The cross-se

11. Rapun Lopez M, Olmedillas H, Pradas de la Fuente $F$, et al. Metabolismo óseo en población infantil y adolescente deportista: Revisión sistemática. Nutr Hosp. 2017;34:1469-81.

12. Drinkwater BL, Nilson K, Chesnut $\mathrm{CH}$, et al. Bone mineral content of amenorrheic and eumenorrheic athletes. N Engl J Med. 1984;311:277-81.

13. Marcus R, Cann C, Madvig P, et al. Menstrual function and bone mass in elite women distance runners. Endocrine and metabolic features. Ann Intern Med. 1985;102:158-63.

14. Johansson J, Nordström A, Nordström P. Objectively measured physical activity is associated with parameters of bone in 70-year-old men and women. Bone. 2015;81:72-9.

15. Landi F, Marzetti E, Martone AM, et al. Exercise as a remedy for sarcopenia. Curr Opin Clin Nutr Metab Care. 2014;17:25-31

16. Kostecka M. The role of healthy diet in the prevention of osteoporosis in perimenopausal period. Pak J Med Sci. 2014:30:763-8.

17. Hibler EA, Sardo Molmenti CL, Dai Q, et al. Physical activity, sedentary behavior, and vitamin D metabolites. Bone. 2016;83:248-55

18. Zhang J, Jameson K, Sayer AA, et al. Accumulation of risk factors associated with poor bone health in older adults. Arch Osteoporos. 2016;11:3

19. Daly RM, Duckham RL, Gianoudis J. Evidence for an interaction between exercise and nutrition for improving bone and muscle health. Curr Osteoporos Rep. 2014;12:219-26.

20. Pasiakos SM, McLellan TM, Lieberman HR. The effects of protein supplements on muscle mass, strength, and aerobic and anaerobic power in healthy adults: A systematic review. Sports Med. 2015;45:111-31. 